\title{
Desafíos para las instituciones de educación superior ante las disrupciones de la Cuarta Revolución Industrial (4IR)
}

\section{Challenges for Higher Education Institutions in addressing the Disruptions of the Fourth Industrial Revolution (4IR)}

\author{
Clavel Maqueda Mireya ${ }^{a}$, Cornejo Velázquez Eduardo $^{b}$ Ovando Chico María Catalina $^{c}$
}

\begin{abstract}
:
The Fourth Industrial Revolution (4IR) is a transformation in the way humans work, socialize and live. The workforce interacts with intelligent technology to solve problems, improve productivity, meet goals and promote competitiveness. 4IR involves developing disciplinary and social skills in the management of organizations. This work described the skills of the academic staff of a mexican public university in reading comprehension, numerical calculation, and problem solving in technology rich environments. Statistical evidence is presented on teacher preference in distance education, and that teachers with higher academic qualifications participate less in formal training activities. Another finding is that there is a need to participate in courses or private classes for specialized disciplinary training topics. In relation to the topics that trigger the 4IR, programming skills and use of statistics or advanced mathematics are not among the capabilities that teachers possess. In addition, it is reported that teachers do not frequently publish scientific literature. The development of superior intellectual skills is still an area of opportunity that requires an intelligent and urgent management strategy.
\end{abstract}

Keywords:

D74 4IR; 123 gestión de la educación; J24 competencias

\section{Resumen:}

La Cuarta Revolución Industrial (4IR) es una transformación en la forma en que los humanos trabajan, socializan y viven. La fuerza de trabajo interactúa con la tecnología inteligente para resolver problemas, mejorar la productividad, cumplir los objetivos y promover la competitividad. 4IR implica desarrollar tanto habilidades disciplinarias como sociales en la gestión de las organizaciones. En este trabajo se describen las aptitudes del personal académico de una universidad pública mexicana en las áreas de comprensión lectora, cálculo numérico y resolución de problemas en entornos ricos en tecnología. Se presenta evidencia estadística sobre la preferencia de los docentes en la formación a distancia y que los docentes con mayor habilitación académica participan menos en actividades formales de capacitación. Otro hallazgo es la necesidad de participar en cursos o clases particulares para temas especializados de formación disciplinar. En relación con los temas que favorecer la adopción de la 4IR, las habilidades de programación y uso de estadística o matemática avanzada no figuran entre las capacidades que utilicen los docentes. Además, se reporta que los docentes no publican frecuentemente sus resultados y experiencias en la literatura científica. El desarrollo de habilidades intelectuales superiores es un área de oportunidad que requiere una estrategia de gestión del entrenamiento y capacitación inteligente y urgente.

\section{Palabras Clave:}

D74 4IR; I23 gestión de la educación; J24 competencias

\section{Introducción}

Según el Informe de Competitividad Global del Foro Económico Mundial (2018), la adaptación de los países a la Cuarta Revolución Industrial (4IR) es un factor determinante en cuanto a su prosperidad o estancamiento (Schwab, 2018). [1]

Los países tienen la oportunidad para aprovechar las nuevas tecnologías (Lee \& Malerba, 2020), con el fin de mejorar la creación de valor económico a través de

\footnotetext{
${ }^{a}$ Autor de Correspondencia, Universidad Popular Autónoma del Estado de Puebla, https://orcid.org/0000-0002-5487-9888, Email: mireya.clavel@upaep.edu.mx

b Universidad Autónoma del Estado de Hidalgo, https://orcid.org/0000-0002-0653-9459, Email: ecornejo@uaeh.edu.mx c Universidad Popular Autónoma del Estado de Puebla, https://orcid.org/0000-0001-5059-2946,

mariacatalina.ovando@upaep.mx
} 
nuevas actividades, mejorar la calidad de los puestos de trabajo en ocupaciones tradicionales y emergentes, y aumentar las habilidades de sus empleados para que alcancen todo su potencial a través de trabajos de alto valor añadido (World Economic Forum, 2018). [2], [3]

El World Economic Forum (2018) estima que para el año 2030 cerca del $14 \%$ de la fuerza laboral mundial deberá adaptarse a la evolución que sufrirá su trabajo como resultado del aumento de la automatización (Schwab, 2019). [4]

Alrededor del $14 \%$ de los trabajadores corre el riesgo de que su trabajo se automatice y el $32 \%$ se enfrentará a cambios significativos en las tareas de su empleo debido a la automatización (Nedelkoska \& Quintini, 2018). La necesidad de interactuar con tecnologías habilitadas con nuevas capacidades requiere que los trabajadores deben tener un mayor nivel de educación y dedicar más tiempo a perfeccionar los aspectos humanos más difíciles de automatizar, por ejemplo, la creatividad y las habilidades emocionales (McKinsey \& Company et al, 2017). [5], [6]

Dadas estas condiciones, es necesario prepararse activamente para que los beneficios potenciales de la 4IR sean aprovechados y al mismo tiempo se puedan subsanar las posibles repercusiones negativas en el sector laboral (Team Smart Industry, 2015). Por ello, es necesario promover un desarrollo más inclusivo que permita el crecimiento de la productividad y competitividad a través de la integración y colaboración entre los sectores público y privado para el desarrollo armonioso entre los beneficios de la sociedad y los avances tecnológicos de la industria (Industry, 2018). [7], [8]

En el informe del Foro Económico Mundial sobre el futuro del trabajo (McKinsey \& Company et al., 2017) se advierte que es necesario subsanar las inconsistencias entre las habilidades actuales y las que se necesitarán para trabajar en el futuro, se requieren estrategias proactivas de gestión de talentos, además de un diálogo y una colaboración continua entre las empresas, gobiernos, proveedores de educación y la sociedad civil (European Commission H2020, 2015). [6], [9]

Ante ello, es imprescindible la construcción de sistemas de educación preparados para el futuro que se ajusten a las nuevas realidades de la 4IR (Deloitte, 2014) donde se desarrollen las habilidades requeridas y exista una conexión entre las necesidades del sector laboral y la instrucción para poder aprovechar el talento global (Tupa, Simota \& Steiner, 2017). [10], [11]
Así, los gobiernos, proveedores de educación y sector privado deberían desarrollar las políticas públicas necesarias para promover oportunidades de aprendizaje y formación para los trabajadores en todas las etapas de sus carreras profesionales.

Las competencias sociales relacionadas con el uso de la tecnología y las no cognitivas están adquiriendo cada vez más importancia (Uhlemann, Lehmann, \& Steinhilper, 2017) y existen importantes oportunidades para que los gobiernos, los empleadores de la industria, los proveedores de educación y otras partes interesadas experimenten e inviertan en nuevas estrategias de educación y formación que serán útiles para los individuos en este nuevo contexto del mercado de trabajo (World Economic Forum, 2018). [12], [3]

Los cambios tecnológicos, las Tecnologías de la Información y las Comunicaciones (TIC) presente en todas las esferas de la vida, junto con los cambios en la estructura del empleo, han dado lugar a una demanda creciente de aptitudes cognitivas de más alto nivel que entrañan la comprensión, la interpretación, el análisis y la comunicación de información compleja (CEPAL, 2018). [13]

Las fuentes de empleo ya no implican tareas cognitivas y manuales rutinarias, sino que se buscan empleados con capacidad para realizar actividades que implican un pensamiento experto y una comunicación compleja.

Las condiciones actuales ejercen una presión para que los empleados desarrollen nuevos y más altos niveles de habilidades, mejorarlas continuamente a lo largo de la vida y usarlas de manera más efectiva (OECD, 2019). Los adultos con mejores aptitudes tienden a tener mayores posibilidades de mantener sus empleos 0 de lograr mejores posiciones y salarios en sus organizaciones. Además, las personas más capacitadas participan más activamente y con mayor confianza en la vida comunitaria y disfrutan de mejor salud (OCDE, 2016). [14], [15]

En necesario diseñar estrategias proactivas de gestión de talentos, enriquecidas mediante el diálogo y colaboración continuos entre las empresas, los gobiernos, los proveedores de educación y la sociedad civil. A medida que el lugar y la forma de los trabajos se transforman, aparecerán nuevos desafíos de gestión y regulación. Por otra parte, es fundamental prepararse para el futuro de un mercado laboral que cambia constantemente para aprovechar las oportunidades y disminuir los efectos negativos (World Economic Forum, 2018). [16] 
En un mundo caracterizado por el cambio acelerado y la incertidumbre, gobiernos, personas, empresas y sindicatos deberán asumir una mayor responsabilidad para garantizar que las personas aprendan y desarrollen sus competencias a lo largo de la vida (Vestberg, 2018). Un mayor compromiso con el aprendizaje protegerá el empleo y la participación de las personas en la sociedad. El desarrollo de un amplio conjunto de conocimientos, competencias, actitudes y valores permitirá que las personas sean trabajadoras competentes y ciudadanas comprometidas (OECD, 2019). [17], [14]

Un mayor compromiso con el aprendizaje protegerá el empleo y la participación de las personas en la sociedad. El desarrollo de un amplio conjunto de conocimientos, competencias, actitudes y valores permitirá que las personas sean trabajadoras competentes y ciudadanas comprometidas (OECD, 2019). [14]

Las organizaciones educativas también requieren la habilitación de sus integrantes para participar en las economías basadas en el conocimiento, beneficiarse de ello y transformar sus procesos internos, puesto que en la medida en que se encuentren preparados, podrán satisfacer las necesidades de formación de la sociedad.

El objetivo de este artículo es describir las competencias clave de los profesores de una universidad pública mexicana para el procesamiento de información en las áreas de comprensión lectora (literacy), cálculo numérico (numeracy) y la solución de problemas (problem solving) con la finalidad de vincularlas con los requerimientos $y$ desafíos de la 4IR.

\section{Estado del arte}

Cuarta Revolución Industrial (4IR)

La 4IR es el resultado de la integración de múltiples "tecnologías exponenciales", como la Inteligencia Artificial (IA), Big Data y Analítica de datos, cómputo en la nube (Cloud Computing), Internet de las Cosas (IoT) y robots autónomos. En esta revolución muchos de los elementos con que se identifica a la industria -fábricas fijas y centralizadas, fuerzas de trabajo masivas dentro de grandes corporaciones- ya no existirán (Penprase, 2018). Ahora dan paso a la Industria 4.0 (14.0) y con ello se reconfiguran las formas de producción, las fuentes de riqueza y el desarrollo en general. [18]

\section{Industria 4.0}

La Industria 4.0 (14.0) es un término utilizado por primera vez en Alemania durante el Hanover Fair 2011, Industrie
4.0 (Kagermann et al., 2011). Es la tríada formada por los objetos físicos, su representación virtual y los servicios y aplicaciones que se añaden a ellos (Fuchs et al., 2019). [19], [20]

14.0 es un metaconcepto para el desarrollo ulterior de los sistemas de producción y creación de valor mediante la vinculación de los mundos real y digital. Describe la vinculación vertical (dentro de la empresa) y horizontal (entre varias divisiones de la empresa o entre varias empresas a lo largo de la cadena de suministro) de estos sistemas ciber-físicos para la producción eficiente, descentralizada y flexible de productos o la prestación de servicios (Bischoff, 2015). [21]

La formación para los adultos es vital para asegurar que aquellos que ya forman parte de la fuerza laboral, y sus empleadores, puedan afrontar los desafíos de la 4IR, mitigar el desempleo, el acceso desigual a recursos y la inactividad (Schwab, 2019). [22]

La capacidad de gestionar la información y resolver problemas en entornos ricos en tecnología se ha convertido en una necesidad a medida que las aplicaciones de las TIC están presentes en los lugares de trabajo, las aulas, las salas de conferencias, los hogares y las interacciones sociales en general (Curtarelli et al., 2016). [23]

Es probable que los adultos que dominan habilidades de este tipo puedan aprovechar al máximo las oportunidades creadas por los cambios tecnológicos y estructurales por los que atraviesan las sociedades modernas. En el caso de la educación superior, los profesores que logren una mejor adaptación y adopción a este nuevo contexto lograrán transferir valiosos conocimientos y experiencias a sus alumnos para contribuir positivamente a su futura vida laboral. Por otro lado, quienes se niegan a utilizar las nuevas tecnologías corren un mayor riesgo ante los inminentes cambios de la 4IR.

\section{Competencias}

El desarrollo de competencias efectivas implica la movilización de conocimiento, competencias, actitudes y valores para satisfacer demandas complejas.

- Competencias básicas, incluyen la comprensión lectora, cálculo numérico y uso de herramientas digitales que están relacionadas con la adaptación a los cambios laborales y sociales. Las personas que cuenten con competencias básicas sólidas estarán mejor posicionadas para adquirir nuevos conocimientos y desarrollar otras competencias: 
- Competencias cognitivas y metacognitivas transversales, el pensamiento crítico, la resolución de problemas complejos, el pensamiento creativo, la competencia de «aprender a aprender» y el autocontrol, son necesarias para mejorar el futuro.

- Competencias sociales y emocionales, la concienciación, la responsabilidad, la empatía, la autosuficiencia y la colaboración.

- Conocimientos y competencias profesionales, técnicas y especializadas, necesarias para satisfacer las demandas de ocupaciones específicas. Las cuales se relacionan con la necesidad de seguir aprendiendo a lo largo de la vida.

En el nuevo contexto, empleadores e industrias proyectan que las aptitudes sociales que incluyen la persuasión, la inteligencia emocional y la capacidad de enseñar a otros serán de gran valor (World Economic Forum, 2016). [24]

La 4IR pone énfasis en la adaptabilidad, en el aprendizaje y el pensamiento autodirigido, ya que la vida útil de cualquier competencia es cada vez más corta y se exige que los trabajadores actualicen continuamente sus habilidades y aprendan por sí mismos nuevas tecnologías y conocimiento disciplinar recientemente desarrollado (Penprase, 2018). [18]

Lo que nos hace humanos, nuestra inteligencia emocional y la creatividad es lo que será demandado. Las mentes adaptables y flexibles serán las más empleables en el futuro, ya que tendrán la agilidad cognitiva para mantenerse al día con el ritmo rápido de cambios.

Accenture (2018) explica que una tendencia importante de la 4IR es la fuerza de trabajo líquida, que se aparta de los antiguos modelos de trabajo que estaban alineados con funciones empresariales específicas (Gleason, 2018). [25]

En este contexto, los docentes universitarios tienen la responsabilidad y compromiso de ser los primeros en adaptarse y desarrollarse en estos escenarios cambiantes, por ello deben aprender e implementar oportunamente y en su propia persona el desarrollo de las competencias básicas.

\section{Estudios relacionados}

Se ha abordado la temática en otras latitudes geográficas, la comunidad científica relacionada con la temática reporta trabajos para identificar los roles de trabajo en las empresas (Benešová \& Tupa, 2017), descripción de las barreras para la adopción de industria
4.0 (Kamble et al., 2018) y la planificación estratégica, las tecnologías clave, las oportunidades y los retos para implementar la Industria 4.0 (Zhou et al., 2016). Otros autores han definido posibles áreas de investigación relacionadas con la introducción del concepto de Industria 4.0 en las PYMES, con especial atención al sector de la construcción (Nowotarski \& Paslawski, 2017). [26], [27], [28], [29]

Así mismo, la literatura científica considera los efectos sobre los lugares de trabajo y las condiciones laborales en las empresas (Reuter et al., 2017) y las necesidades para implementar la Industria 4.0 (Knolle, 2016; Dalenogare et al., 2018; Felix \& Rosa, 2018). [30], [31], [32], [33]

En otros artículos se describe el proceso de transformación de un taller de fabricación dirigido a la formación para contribuir a facilitar el camino hacia nuevas tendencias de fabricación como la 14.0 en un contexto académico que fortalezca el proceso de formación de ingenieros (Baena et al., 2017). [34]

Por otra parte, se describen los impactos de la Industria 4.0 en la educación superior y su importancia en la educación a través del análisis estadístico de datos (Baygin et al., 2016; Olmos, 2017). [35], [36]

En México la Secretaría de Economía publicó el reporte "Crafting the future a roadmap for Industry 4.0 in México" pero es un planteamiento a futuro y se adolece de estudios que diagnostiquen nuestra situación y puedan reportar un perfil del empleado en los entornos de la 4IR (SE, 2016). [37]

\section{Desarrollo}

Para el presente trabajo se realizó un estudio transversal de tipo empírico basado en la aplicación de la encuesta de campo para un posterior análisis fundamentado en un proceso inductivo. Aplicamos el cuestionario a una muestra de docentes de una organización educativa pública mexicana en el mes de enero del año 2020 mediante la herramienta de Google Forms.

El procesamiento de la información se implementó por etapas, iniciando con la recolección de los datos por medio de los cuestionarios que fueron distribuidos por correo electrónico, en la segunda etapa se lleva a cabo el análisis estadístico con la herramienta STATA versión 13 donde se utilizaron técnicas de estadística descriptiva para realizar el análisis de las variables de interés. 
Se utilizó la ecuación para el cálculo del tamaño de la muestra para una población finita (Moguel, 2005) con un nivel de confianza del $99 \%$ y un error muestral del $5 \%$, para una población de 4,730 docentes. Los parámetros adicionales de la ecuación fueron: $p=69 \%$ (nivel de prevalencia) y $q=31 \%$ (nivel de fracaso). El tamaño calculado para la muestra fue de 507 sujetos de estudio. [38]

La selección de los elementos de la muestra se hizo de forma aleatoria de los profesores que imparten clases en los niveles de bachillerato, licenciatura y posgrado.

Se utilizó el cuestionario para la Evaluación Internacional de las Competencias de los Adultos (PIAAC) del Programa de la Organización para la Cooperación y el Desarrollo Económico (OCDE) como instrumento de recolección de información (OCDE, 2019). [14]

PIAAC proporciona información sobre el grado en que las personas adultas pueden desempeñar las aptitudes fundamentales que necesita la sociedad, y sobre la forma en que las utilizan en el trabajo y en el hogar. En ella se evalúa la competencia de los adultos en tres aptitudes fundamentales para el procesamiento de la información: comprensión lectora, cálculo numérico y resolución de problemas en entornos ricos en tecnología.

Las aptitudes de comprensión lectora y cálculo numérico constituyen una base para desarrollar habilidades cognitivas de orden superior y son esenciales para acceder y comprender dominios específicos de conocimiento. Además, son pertinentes en toda una serie de contextos de la vida, desde la educación, el trabajo, el hogar, la vida social y la interacción con las autoridades.

En el marco del PIAAC, se entiende por comprensión lectora el "comprender, evaluar, utilizar y vincularse con el texto escrito para participar en la sociedad, alcanzar los objetivos propios y desarrollar los conocimientos y el potencial propios". El cálculo numérico se define como "la capacidad de acceder, utilizar, interpretar y comunicar información e ideas matemáticas, para participar y gestionar las demandas matemáticas de una serie de situaciones en la vida adulta". La resolución de problemas en entornos ricos en tecnología es entendida como "el uso de la tecnología digital, las herramientas de comunicación y las redes para adquirir y evaluar información, comunicarse con otros y realizar tareas prácticas" (Ortiz, 2014). [39]

\section{Resultados}

El instrumento recolectó información de las actividades de aprendizaje formal desarrolladas durante los 12 meses anteriores a la aplicación de la encuesta (año 2019). Permite identificar las diferencias entre los subgrupos de la muestra con relación al último nivel de estudios reportado por los profesores que se presentan en la Tabla 1.

\begin{tabular}{l|rrr} 
Último nivel de estudio & Frecuencia & Porcentaje & Acumulado \\
\hline Licenciatura & 125 & 24.65 & 24.65 \\
Especialidad & 46 & 9.08 & 33.73 \\
Maestria & 163 & 32.15 & 65.88 \\
Doctorado & 147 & 28.99 & 94.87 \\
Posdoctorado & 26 & 5.13 & 100 \\
\hline Total & 507 & 100 & \\
\hline
\end{tabular}

Tabla 1. Grupos en la muestra por el último nivel de estudios.

Los profesores con posgrado representan el $66.27 \%$ de la muestra. El subgrupo con grado de maestría es el más grande y se forma con 163 profesores, 32.15\%. Los subgrupos de profesores que tienen doctorado son 147, $28.99 \%$, con licenciatura son $125,24.65 \%$, con especialidad son $46,9.08 \%$, y con posdoctorado son 26 , $5.13 \%$.

El $91.46 \%$ de los profesores encuestados respondieron que durante el año 2019 participaron en actividades de entrenamiento o capacitación formal. Los principales motivos para participar en estas actividades son hacer mejor su trabajo y/o mejorar sus perspectivas profesionales, $77.56 \%$, y aumentar su conocimiento o habilidades en temas de su interés, $18.44 \%$.

El análisis de la participación en actividades de capacitación o entrenamiento formal se presentan en la Tabla 2. El $49.11 \%$ de los profesores reportaron que participaron en cursos de educación abierta o a distancia; el $19.72 \%$ en sesiones de capacitación en el puesto de trabajo o impartida por sus supervisores o compañeros de trabajo y $13.21 \%$ en seminario o taller. Resalta que el $40.3 \%$ de los docentes que reportaron participación en seminarios tiene el grado de doctor. 


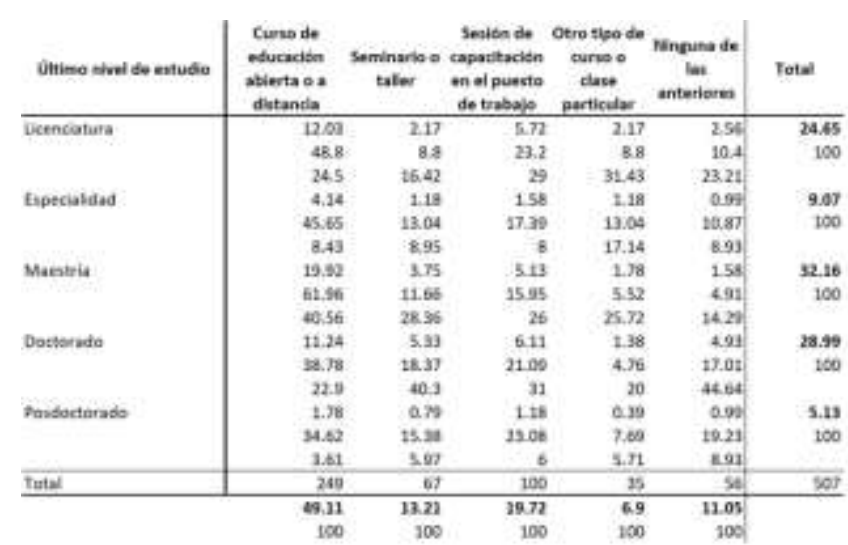

Tabla 2. Actividades de entrenamiento formal por nivel de estudio.

El $11.05 \%$ de los encuestados respondió que no realizó actividades de capacitación y el subgrupo más representativo es el de los profesores con grado de doctorado, $44.64 \%$. Los docentes con grado de maestría representan la mayor proporción de participantes en actividades de capacitación formal, $32.16 \%$, el siguiente subgrupo corresponde a los docentes con grado de doctor, $28.99 \%$ y en tercer sitio, los docentes con licenciatura, $24.65 \%$.

Los profesores con grado de maestría tienen una participación en curso de educación abierta o a distancia, de $61.96 \%, 10$ puntos porcentuales más que la proporción general. Además, representan el $40.56 \%$ de todos los participantes en cursos de educación abierta o a distancia.

De acuerdo con los datos muestrales e intergrupales de media presentados en la Tabla 3 los cursos de educación abierta o a distancia son la actividad de capacitación o entrenamiento formal con el mayor número de participaciones reportadas con media $=2.108481$.

\begin{tabular}{l|rrrrr} 
Último nivel de estudio & $\begin{array}{r}\text { Cursos de } \\
\text { educación } \\
\text { abierta o a } \\
\text { distancia }\end{array}$ & $\begin{array}{c}\text { Sesión de } \\
\text { en el puesto } \\
\text { de trabajo }\end{array}$ & $\begin{array}{c}\text { Seminario o } \\
\text { taller }\end{array}$ & $\begin{array}{c}\text { Curso o } \\
\text { clase } \\
\text { particular }\end{array}$ \\
\hline Licenciatura & 2.352 & 2.128 & 1.408 & 1.024 \\
Especialidad & 1.956522 & 1.73913 & 1.391304 & 0.9782609 \\
Maestria & 2.245399 & 1.852761 & 1.852761 & 0.8895706 \\
Doctorado & 1.891156 & 1.52381 & 1.884354 & 0.7482993 \\
Posdoctorado & 1.576923 & 1.615385 & 2.230769 & 0.5 \\
\hline Total & 2.108481 & 1.802761 & 1.729783 & 0.8698225 \\
\hline
\end{tabular}

Tabla 3. Actividades de entrenamiento formal por nivel de estudio.

El segundo grupo de actividades el mayor número de participaciones corresponde a las sesiones de capacitación organizadas en el puesto de trabajo impartidas por sus supervisores o compañeros de trabajo, media $=1.802761$. La participación en seminarios 0 talleres es la actividad en el tercer nivel de importancia con una media $=1.729783$. El cuarto grupo de actividades corresponde a la participación en curso o clase particulares con una media de 0.8698225 .

El análisis de los datos de la Tabla 4 corresponden con las actividades de comprensión lectora en el trabajo reportadas por los profesores.

\begin{tabular}{|c|c|c|c|c|c|}
\hline Compranuión lectora & Nunca & $\begin{array}{c}\text { Menos de } \\
\text { una ver al } \\
\text { mes }\end{array}$ & $\begin{array}{c}\text { Menos de } \\
\text { una vez a la } \\
\text { semanas } \\
\text { pero al } \\
\text { menos una } \\
\text { ver al mes }\end{array}$ & $\begin{array}{l}\text { Al menos } \\
\text { una vez a ls } \\
\text { sernana, } \\
\text { pero no } \\
\text { todos los } \\
\text { dias }\end{array}$ & $\begin{array}{c}\text { Todos los } \\
\text { dias }\end{array}$ \\
\hline Leer indicaciones & 0.79 & 5.33 & 8.09 & 27,81 & 57.99 \\
\hline Leer cartas & 0.39 & 0.39 & 1.38 & 15.19 & 82.45 \\
\hline Leer articulos & 1.18 & 5.52 & 8.09 & 43.59 & 41.52 \\
\hline Leer laros & 0.79 & 9.27 & 17,16 & 36.29 & 36.49 \\
\hline Leer mantaales: & 1.58 & 7.89 & 18,74 & 42.8 & 28.99 \\
\hline Leer recibas & 8.88 & 21.5 & 35.31 & 25.84 & 8.48 \\
\hline Leer diagramas & 2.96 & 11.05 & 17.36 & 38.65 & 29.98 \\
\hline Escribir cartas & 0.39 & 3.55 & 4.73 & 29.59 & 61.74 \\
\hline Escribir articulos & 35.31 & 34.12 & 10.45 & 10.26 & 9.86 \\
\hline Escribir informes & 6.51 & 33.53 & 33.53 & 19.72 & 6.71 \\
\hline Escribir formatos & 2.37 & 20.71 & 25.05 & 28.6 & 23.27 \\
\hline
\end{tabular}

Tabla 4. Realización de actividades de comprensión lectora.

Los resultados describen que las actividades de comprensión lectora más frecuentes en el trabajo son lectura de cartas, notas o correos electrónicos, $82.45 \%$, leer indicaciones o instrucciones, $57.99 \%$ y escribir cartas, notas o correos electrónicos, $61.74 \%$. Mientras que las tres actividades menos reportadas son escribir artículos para periódicos, revistas o boletines de noticias, $69.43 \%$, escribir informes, $40.04 \%$, y escribir en formatos, $23.08 \%$.

Los datos de la Tabla 5 corresponden con la realización de actividades de cálculo numérico en el trabajo por parte de los profesores.

\begin{tabular}{|c|c|c|c|c|c|}
\hline Calculo numérico & Nunca & $\begin{array}{c}\text { Menos de } \\
\text { una vez al } \\
\text { mes }\end{array}$ & $\begin{array}{c}\text { Menos de } \\
\text { una vez a } \\
\text { la semana, } \\
\text { pero al } \\
\text { menas una } \\
\text { ver al mes }\end{array}$ & $\begin{array}{c}\text { Al menas } \\
\text { uns vers a } \\
\text { lo semans. } \\
\text { pero no } \\
\text { todas los } \\
\text { dias }\end{array}$ & $\begin{array}{c}\text { Todes los } \\
\text { dilas }\end{array}$ \\
\hline Precias & 23.47 & 31.36 & 15.78 & 17.75 & 11.64 \\
\hline Fracoiones & 11.44 & 215 & 17.75 & 26,82 & 22.49 \\
\hline Calculadora & 3.94 & 10.45 & 17,36 & 36.09 & 32.15 \\
\hline Graficoss & 4.93 & $15.5 \%$ & 17.55 & 41.62 & 20,32 \\
\hline Álgetirs & 13.61 & 24.26 & 11.83 & 26.23 & 24.06 \\
\hline Estadistica & 30.97 & 213 & 11.64 & 17.95 & 18.15 \\
\hline
\end{tabular}

Tabla 5. Realización de actividades de cálculo numérico.

Las actividades con cálculos numéricos que se realizan todos los días en el trabajo son el uso de la calculadora de mano o en la computadora, $32.15 \%$, y el uso de álgebra simple o fórmulas, $24.06 \%$. Por otro lado, las actividades de cálculo numérico que nunca se realizan son el uso de estadística o matemática avanzadas, como cálculo, álgebra compleja, trigonometría o análisis de 
regresión, 30.97\%, y el cálculo de precios, costos o presupuestos, $23.47 \%$.

La frecuencia de uso de herramientas TIC en el trabajo se presenta en la Tabla 6. Los profesores usan todos los días Internet, 85.8\%, correo electrónico, $84.62 \%$ y procesador de textos, $76.13 \%$.

\begin{tabular}{|c|c|c|c|c|c|}
\hline $\begin{array}{l}\text { Interacción can las } \\
\text { Tecnologáa de la } \\
\text { Información }\end{array}$ & Nunca & $\begin{array}{l}\text { Menos de } \\
\text { una vez al } \\
\text { mes }\end{array}$ & $\begin{array}{c}\text { Menos de } \\
\text { una ver a la } \\
\text { semana, } \\
\text { pero al } \\
\text { menos una } \\
\text { ver al mes }\end{array}$ & $\begin{array}{c}\text { Al menos } \\
\text { uns vex a ls } \\
\text { semana, } \\
\text { pero no } \\
\text { todos los } \\
\text { dias }\end{array}$ & $\begin{array}{c}\text { Todos les } \\
\text { dias }\end{array}$ \\
\hline Email & 0 & 0.59 & 1.58 & 13.21 & 84.62 \\
\hline internet & 0.39 & 0.79 & 2.96 & 10.06 & 85.8 \\
\hline Transacción & 22.88 & 25.64 & 24.26 & 19.53 & 7.69 \\
\hline Hojaçáloula & 1.97 & 11.64 & 14.2 & 34,71 & 37.48 \\
\hline Procésadar & 0.2 & 0.79 & 2.56 & 20.32 & 76.13 \\
\hline Programación & 53.25 & 20.51 & 7,3 & 828 & 10.65 \\
\hline Comanicar & 14,79 & 30.77 & 16.37 & 18.34 & 19.72 \\
\hline
\end{tabular}

Tabla 6. Interacción con las Tecnología de la Información (TIC).

Las herramientas TIC que nunca se utilizan son lenguaje de programación para programar o escribir código de computadora, 53.25\%, transacciones en Internet para comprar o vender productos o servicios o realizar operaciones bancarias, $22.88 \%$, y conversaciones en tiempo real usando Internet, $14.79 \%$.

Los resultados de las habilidades en el procesamiento de información para resolución de problemas se presentan en la Tabla 7.

\begin{tabular}{|c|c|c|c|c|c|}
\hline $\begin{array}{l}\text { Resolutión de problemas } \\
\text { en entornos ricos en } \\
\text { tecnologis }\end{array}$ & Nuncs & $\begin{array}{l}\text { Mences de } \\
\text { una vez al } \\
\text { mes }\end{array}$ & $\begin{array}{c}\text { Menos de } \\
\text { una ver a la } \\
\text { semana, } \\
\text { pero al } \\
\text { menos una } \\
\text { vec al mes }\end{array}$ & $\begin{array}{c}\text { Al menes } \\
\text { una vez a la } \\
\text { semans, } \\
\text { pero no } \\
\text { todos los } \\
\text { diss }\end{array}$ & $\begin{array}{l}\text { Todos los } \\
\text { días }\end{array}$ \\
\hline Aplicar & 0 & 2.56 & 18.93 & 36.49 & $\$ 2.01$ \\
\hline Aprender: & 0 & 0.39 & 4.14 & 19.33 & 76.13 \\
\hline Relacionar & 0 & 0.39 & 9.86 & 30.18 & 59.57 \\
\hline Diticiles & 0.39 & 0.59 & 0.27 & 38.46 & 51.28 \\
\hline Entender & 0.39 & 0.79 & 13.21 & 33.53 & 52.07 \\
\hline Buscar & 0.2 & 0.59 & 5.13 & 20.51 & 33.57 \\
\hline
\end{tabular}

Tabla 7. Resolución de problemas.

Los profesores contestaron que están de acuerdo en gran medida y totalmente con relacionar nuevas ideas con situaciones de la vida real en las se podrían aplicar, el aprender nuevas cosas, $76.13 \%$, relacionar algo nuevo con lo que ya saben, 59.57\% llegar hasta el fondo de las cosas difíciles, entender cómo encajan las ideas diferentes $52.07 \%$ y la búsqueda de información para aclarar algo que no entiende, $73.57 \%$.

\section{Conclusiones y discusión}

Los resultados de la Tabla 2 describen que los docentes prefieren las actividades de capacitación en educación abierta o a distancia, pero se involucran también en actividades de formación para el puesto de trabajo que promueve la institución.

Los docentes con mayor habilitación académica se involucran en menor medida en las actividades de capacitación o entrenamiento formal (44\% del total que respondieron participar en ninguna actividad de capacitación formal) y evidencia que se vinculan mayormente en actividades inherentes a sus actividades profesionales (seminarios o talleres). Esta situación no coincide con los reportes de la OCDE que sugieren que la participación en la actualización es preferida por los individuos con mayor grado de formación (OCDE, 2019).

Los resultados de la Tabla 3 describen que los profesores con licenciatura (media $=2.352$ ) y maestría (media $=$ 2.245399) participan en cursos de educación abierta o a distancia por encima de la media muestral. Los profesores con maestría (media $=1.852761$ ) participan en sesiones de capacitación organizadas en el puesto de trabajo impartidas por sus supervisores o compañeros de trabajo por encima de la media muestral.

En el caso de la participación en seminarios o talleres los profesores con maestría (media $=1.852761$ ), doctorado $($ media $=1.884354)$ y posdoctorado $($ media $=2.230769)$ están por encima de la media muestral. Los profesores con especialidad (media $=0.9782609$ ) y maestría (media $=0.8895706$ ) participan en actividades curso o clase particulares por encima de la media muestral.

La distribución descrita sugiere revisar las ofertas de actualización en temáticas disciplinares especializadas, debido a la evidencia relacionada con la necesidad de elegir participar en cursos o clases particulares, en la que el costo está asociado a la calidad y actualidad.

Los resultados presentados en la Tabla 4 describen que los profesores realizan actividades de lectura de documentos todos los días o al menos una vez a la semana. Respecto de las actividades de escritura de documentos un alto porcentaje de profesores nunca o menos de una vez al mes escribe artículos para periódicos, revistas o boletines de noticias, informes y formatos.

La Tabla 5 describe que las actividades de cálculo numérico que realizan todos los días o al menos una vez a la semana son el uso de la calculadora de mano o en la computadora, uso de álgebra simple o fórmulas, elaborar esquemas, gráficos 0 tablas. Por otra parte, las actividades que nunca o menos de una vez al mes realizan son el uso de estadística o matemática 
avanzadas, como cálculo, álgebra compleja, trigonometría o análisis de regresión y el cálculo de precios, costos o presupuestos.

De acuerdo con los resultados de la Tabla 6, las herramientas TIC que más utilizan los profesores universitarios son Internet, correo electrónico y procesador de texto. Por otro lado, las que menos se utilizan son lenguajes de programación para programar o escribir código de computadora, transacciones en Internet para comprar o vender productos o servicios 0 realizar operaciones bancarias y conversaciones en tiempo real usando Internet.

Los temas que detonan la 4IR están íntimamente vinculados con el uso y aplicación de nuevas tecnologías (loT, cloud computing, robótica, simulación, tecnologías móviles) que requiere que las habilidades de programación y el uso de uso de estadística o matemática avanzada en todos los niveles (IA, big data y analítica de datos) debería prevalecer entre las capacidades que se poseen sobre todo en los niveles de habilitación superiores.

Los hallazgos del presente trabajo sugieren que se cuenta con un capital humano con habilidades básicas, talento y motivación, pero el desarrollo de habilidades intelectuales superiores es un área de oportunidad que requiere una estrategia de gestión inteligente y urgente.

El que se reporte que no se escribe y difunde el conocimiento generado al interior de la organización a través de la publicación de literatura científica, es una llamada de alerta, por tratarse de actividades sustantivas y esenciales en una institución de educación superior.

Finalmente, se recomienda generar una estrategia de formación continua y para afrontar la transición de la 4IR, utilizando medios tecnológicos, vinculado con organismos e instituciones con experiencia que promuevan la colaboración y asociado a la mejora de las perspectivas profesionales, lo anterior es urgente, puesto que las revoluciones industriales anteriores han demostrado que los cambios traen oportunidades para quienes se adaptan rápidamente a las nuevas condiciones y evolucionan.

\section{Referencias}

[1] Schwab. K. (2018). The global competitiveness report. World Economic Forum (Vol. 5). https://doi.org/92-95044-35-5
[2] Lee, K., Malerba, F., \& Primi, A. (2020). The fourth industrial revolution, changing global value chains and industrial upgrading in emerging economies. Journal of Economic Policy Reform, 1-12.

[3] World Economic Forum. (2018). The Future of Jobs Report 2018 Insight Report Centre for the New Economy and Society. https://doi.org/10.1177/1946756712473437

[4] Schwab K. (2019). The Global Competitiveness Report 2019. Genova: World Economic Forum.

[5] Nedelkoska, L., \& Quintini, G. (2018). Automation, skills use and training. In OECD Social, Employment and Migration Working Papers. OECD Publishing, París. doi:http://dx.doi. org/10.1787/2e2f4eea-en

[6] McKinsey \& Company, Manyika, J., Chui, M., Miremadi, M., Bughin, J., George, K., ... Dewhurst, M. (2017). A future that works: Automation, employment, and productivity. Mckinsey Global Institute, (January), 148. https://doi.org/10.1109/MMSE.2002.1181615

[7] Team Smart Industry. (2015). Action Agenda Smart Industry The Netherlands, 5.

[8] Industry, S. (2018). Smart Industry implementation Agenda 20182021.

[9] European Commission H2020. (2015). A Guide to ICT-related activities in WP2016-17. Pp, 1-12.

[10]Deloitte Consulting, L. L. P., \& by Deloitte, B. (2014). Global human capital trends 2014: Engaging the 21st century workforce.

[11] Tupa, J., Simota, J., \& Steiner, F. (2017). Aspects of risk management implementation for Industry 4.0. Procedia Manufacturing, 11, 12231230.

[12] Uhlemann, T. H. J., Lehmann, C., \& Steinhilper, R. (2017). The digital twin: Realizing the cyber-physical production system for industry 4.0 . Procedia Cirp, 61, 335-340.

[13]CEPAL. (2018). Monitoreo de la Agenda Digital para América Latina $\begin{array}{llll}\text { y el Caribe } & \text { eLAC2018, }\end{array}$ https://repositorio.cepal.org/bitstream/handle/11362/43444/1/S180025 6_es.pdf

[14] Organization for Economic Co-operation and Development (OECD). (2019). OECD Skills Strategy 2019: skills to shape a better future. OECD, Paris, France.

[15] Organization for Economic Co-operation and Development (OECD). (2016). La naturaleza del aprendizaje: Usando la investigación para inspirar la práctica. Entornos, 29(2), 467-468.

[16] World Economic Forum (2018). La cuarta revolución industrial, November 23, 2018, https://toplink.weforum.org/knowledge/insight/a1Gb0000001RIhBEA W/explore/summary

[17] Vestberg, H. (2018). Why we need both science and humanities for a Fourth Industrial Revolution education. https://europeansting.com/2018/09/24/why-we-need-both-scienceand-humanities-for-a-fourth-industrial-revolution-education/

[18]Penprase B.E. (2018) The Fourth Industrial Revolution and Higher Education. In: Gleason N. (eds) Higher Education in the Era of the Fourth Industrial Revolution. Palgrave Macmillan, Singapore. https://doi.org/10.1007/978-981-13-0194-0_9 [34] Baena, F., Guarin, A., Mora, J., Sauza, J., \& Retat, S. (2017). Learning Factory: The Path 
to Industry 4.0. Procedia Manufacturing, 9, 73-80. https://doi.org/10.1016/j.promfg.2017.04.022

[19] Kagermann H., Lukas, W. \& Wahlster, W. (2011). "Industrie 4.0: Mit dem Internet der Dinge auf dem Weg zur 4. industriellen Revolution," VDI nachrichten

[20]Fuchs, J., Schmidt, J., Franke, J., Rehman, K., Sauer, M., \& Karnouskos, S. (2019, September). I4. 0-compliant integration of assets utilizing the Asset Administration Shell. 24th IEEE International Conference on Emerging Technologies and Factory Automation (ETFA) (pp. 1243-1247). IEEE.

[21]Bischoff, J. (2015). Erschließen der Potenzialeder Anwendung von Industrie 4.0 im Mittelstand. Bundesministerium für Wirtschaft und Energie (BMWi). https://www.bmwi.de/Redaktion/DE/Publikationen/Studien/erschliess en-der-potenziale-der-anwendung-von-industrie-4-0-immittelstand.pdf\%3F_blob\%3DpublicationFile\%26v\%3D5

[22] Schwab, K. (2019, December). Manifesto de Davos 2020: El propósito universal de las empresas en la Cuarta Revolución Industrial. In World Economic Forum (Vol. 1, pp. 1-4).

[23]Curtarelli, M., Gualtieri, V., Jannati, M. S., \& Donlevy, V. (2016). ICT for work: Digital skills in the workplace. http://knjiznica.sabor.hr/pdf/E_publikacije/ICT\%20for\%20work.pdf

[24]World Economic Forum. (2016, January). The future of jobs: Employment, skills and workforce strategy for the fourth industrial revolution. In Global challenge insight report. Geneva: World Economic Forum.

[25] Gleason, N. (2018) N. W. Gleason (ed.), Higher Education in the Era of the Fourth Industrial Revolution, https://doi.org/10.1007/978-98113-0194-0_7

[26]Benešová, A., \& Tupa, J. (2017). Requirements for Education and Qualification of People in Industry 4.0. Procedia Manufacturing, 11(June), 2195-2202. https://doi.org/10.1016/j.promfg.2017.07.366

[27] Kamble, S. S., Gunasekaran, A., \& Sharma, R. (2018). Analysis of the driving and dependence power of barriers to adopt industry 4.0 in Indian manufacturing industry. Computers in Industry, 101(May), $107-$ 119. https://doi.org/10.1016/j.compind.2018.06.004

[28]Zhou, K., Liu, T., \& Zhou, L. (2016). Industry 4.0: Towards future industrial opportunities and challenges. 2015 12th International Conference on Fuzzy Systems and Knowledge Discovery, FSKD 2015 , 2147-2152. https://doi.org/10.1109/FSKD.2015.7382284

[29] Nowotarski, P., \& Paslawski, J. (2017). Industry 4.0 Concept Introduction into Construction SMEs. IOP Conference Series: Materials Science and Engineering, 245(5). https://doi.org/10.1088/1757-899X/245/5/052043

[30]Reuter, M., Oberc, H., Wannöffel, M., Kreimeier, D., Klippert, J., Pawlicki, P., \& Kuhlenkötter, B. (2017). Learning Factories' Trainings as an Enabler of Proactive Workers' Participation Regarding Industrie 4.0. Procedia Manufacturing, 9, 354-360. https://doi.org/10.1016/j.promfg.2017.04.020

[31]Knolle, K. (2016). Pervichnoe Utolshchenie 'Endokarda V Rannem Detskom Vozraste. Arkhiv Patologii, 25(25), 60-64. https://doi.org/10.1016/j.ifacol.2016.12.002

[32]Dalenogare, L. S., Benitez, G. B., Ayala, N. F., \& Frank, A. G. (2018) The expected contribution of Industry 4.0 technologies for industrial performance. International Journal of Production Economics, 204(August), 383-394. https://doi.org/10.1016/j.ijpe.2018.08.019
[33]Felix, A., \& Rosa, E. (2018). a Gestão Documental Como Suporte Ao Governo Eletrônico : Caso Da Secretaria De Estado Da Saúde De Santa Catarina ( Ses / Sc ). https://doi.org/10.1561/2200000016

[34]Baena, F., Guarin, A., Mora, J., Sauza, J., \& Retat, S. (2017). Learning Factory: The Path to Industry 4.0. Procedia Manufacturing, 9, 73-80. https://doi.org/10.1016/j.promfg.2017.04.022

[35]Baygin, M., Yetis, H., Karakose, M., \& Akin, E. (2016). An effect analysis of industry 4.0 to higher education. 2016 15th International Conference on Information Technology Based Higher Education and Training, ITHET 2016. https://doi.org/10.1109/ITHET.2016.7760744

[36] Olmos, D. (2017). Big Data y el consumo energético en la Industria 4.0 y las Smart Cities. Agenda de la empresa andaluza: ideas, personas e instrumentos para dirigir la empresa, (224), 69.

[37] Secretaría de Economía de México SE. (2016). Crafting the future: A roadmap for industry 4.0 in Mexico.

[38]Moguel, E. A. R. (2005). Metodología de la investigación. Univ. J. Autónoma de Tabasco.

[39] Ortiz, A. (2014). Competencia matemática a lo largo de la vida y educación de adultos. (Programa para la Evaluación Internacional de las Competencias de los Adultos: PIAAC). 